\title{
EDITORIAL
}

\section{World Molecular Imaging Congress 2015: Precision Medicine Visualized}

\author{
H. Charles Manning
}

Institute of Imaging Science and Center for Molecular Probes, Vanderbilt University Medical Center, Nashville, TN, 37232, USA

I am delighted on this occasion to alert the readers of Molecular Imaging and Biology of the upcoming 8th annual meeting of the World Molecular Imaging Congress (WMIC). This year's congress will be held September 2-5, 2015 in beautiful Honolulu, Hawaii. Known historically as the "Crossroads of the Pacific," Honolulu is an exceptional venue for our congress where an incredibly diverse repertoire of imaging scientists and clinicians will assemble to share the latest and most exciting discoveries in molecular imaging in a relaxing, friendly atmosphere. Early abstract submission has far-exceeded expectations, boding incredibly well for the excitement certain to epitomize our meeting this year. In addition, I am pleased to remind you that LateBreaking Abstracts will be accepted June 1-10, 2015; so as you read this, it is likely that time still remains to submit a scientific abstract for the meeting.

The theme of the 2015 meeting is Precision Medicine, Visualized, which embodies current and future roles of molecular imaging in basic science, translational medicine, and healthcare. Precision medicine is an emerging approach for disease treatment and prevention that takes into account individual variability in genes, environment, and lifestyle for each person. While significant advances in precision medicine have been made for certain cancers, the practice is not commonplace for most diseases. Many efforts are now underway to help make precision medicine the norm rather than the exception. To accelerate the pace, you are likely aware that President Obama recently unveiled a Precision Medicine Initiative in the United States-a bold step towards moving the concept of precision medicine into every day clinical practice. As our members know, molecular imaging excels at visualizing and quantifying the cellular and molecular underpinnings of diseases on a patient-by-patient basis and provides new ways to detect disease at early, potentially curable stages. Molecular imaging may also be used to identify patients likely to respond to certain treatments and predict response to therapy very early, prior to conventional means. As such, molecular

Correspondence to: H. Manning; e-mail: henry.c.manning@vanderbilt.edu imaging has tremendous potential to advance precision medicine in many critical human diseases and may reduce the overall cost of healthcare. As a society, we recognize that the future success of precision medicine will depend upon molecular imaging.

As the world's leading forum for the presentation of groundbreaking research in molecular imaging, probe chemistry, and quantitative biology, WMIC 2015 will feature the newest discoveries certain to guide precision medicine initiatives. Exemplifying the tremendous creativity brought to molecular imaging by World Molecular Imaging Society (WMIS) member scientists and clinicians from many disciplines and from around the world, the 2015 meeting is certain to be a memorable and transformative occasion.

Our program this year is comprised of five major categorical emphases: Chemistry and Imaging Probes, First-in-Human and Clinical Studies, Preclinical Cell and Tissue Level Studies, Preclinical in vivo Studies and Technology and Software Developments. These emphases are further distilled into more focused categories that elaborate a fully comprehensive program.

Building upon prior meeting successes and member feedback, we are pleased to illuminate a number of "firsts" associated with the 2015 WMIC Program. These include a new, streamlined format for oral sessions, which will include a "Highlights" award lecture selected from the highestscoring scientific abstracts, followed by six focused talks in rapid succession. Several new program elements specifically target future aspects of molecular imaging, including formal recognition of abstracts that exemplify the "Hot Topics" defined by the Society and the first WMIC Fellow's Forum, where luminaries in the field will share their visions for future directions in molecular imaging in an informal and interactive setting.

Several program elements have been envisioned to connect attendees with newly commercialized molecular imaging technologies. New in 2015, scientific abstracts are being solicited for a special "exhibitors-only" track; each submitted abstract will be reviewed, scored, and included in meeting materials. The highest-scoring exhibitor abstracts 
will be featured in a special oral session, following which WMIS members will select one finalist for a new exhibitor award, "Commercial Innovation of the Year".

We have gathered a group of internationally renowned plenary speakers including Zena Werb, Chien Ho, Swati Piramal, Philipp Scherer, Jon-Kar Zubieta, and Elizabeth Morris. These outstanding speakers from around the globe will speak on a diversity of broadly appealing topics that include metabolism, immunology, neuroscience, and cancer. In addition, John Gore will deliver the ever-popular Meeting Highlights Lecture, where the most exciting science presented throughout this year's Congress will be summarized in a single summary lecture. In addition to outstanding plenary lectures, this year's program features an incredible collection of spotlight sessions that lead off each day of the scientific program. Topics of this year's spotlight sessions include infectious disease, men's and women's imaging, ultrasound, companion diagnostics, metabolism, radiogenomics, and early career. Beyond these sessions, industry leaders from major pharmaceutical companies will hold a panel discussion on critical barriers affecting translational imaging and drug discovery.

In 2014, the WMIC completed a 3-year education program cycle. Building upon this widely popular aspect of the WMIC, in 2015, we are pleased to introduce an entirely new education program, featuring several new tracks while retaining some of most popular from previous years. The education co-chairs for 2015 are Kimberly Kelly and Danielle Vughts; they have assembled an outstanding program certain to be valuable for all investigators, seasoned and new. This year's education program will launch with a special "Molecular Imaging-101" lecture delivered by John Frangioni, which immediately precede four parallel tracks that explore molecular imaging principles and practices (CME), molecular imaging in pharmaceutical discovery, development, and toxicity, hot topics in molecular imaging, and professional development. In 2015, we are pleased to offer continuing medical education tracks each day of the meeting.

Our Poster Program Chair Jan Grimm and his committee have developed a stimulating and highly interactive Poster Recognition Program. As always, submissions will be reviewed by members of the program committee and awards will be given to the best posters based on scientific content and quality of presentation. New to 2015, award-winning posters will also be featured at select times in a PowerPresentation Theater located in the Exhibit Hall-you won't want to miss this opportunity to meet the award winners and hear more about their innovative science. Furthermore, in 2015, exhibitors will have the opportunity to select an abstract from the poster program that illustrates the most innovative utilization of their technology; these awardwinning abstracts will be highlighted in two special poster sessions during the week.

In summary, I sincerely hope that you take part in the 2015 WMIC meeting, where we will Discover, Visualize, Learn, and Cure with molecular imaging as an international society. I look forward to seeing you in Honolulu.

Conflict of Interest. The author declares no conflict of interest. 